\title{
Adaptability of Organic Matter and Solid Content to Fe2+/Persulfate and Skeleton Builder Conditioner for Waste Activated Sludge Dewatering
}

\section{Xiaoran Li}

Hubei University of Technology

Yafei Shi ( $\nabla$ yfshi@hbut.edu.cn )

Hubei University of Technology https://orcid.org/0000-0001-7713-0813

\section{Xi Zhou}

Hubei University of Technology

Lu Wang

Hubei University of Technology

\section{Huiqin Zhang}

Hubei University of Technology

Kewu Pi

Hubei University of Technology

Andrea R Gerson

Blue Minerals Consultancy

Defu Liu

Hubei University of Technology

\section{Research Article}

Keywords: Sewage sludge, Conditioning, Persulfate oxidation, Skeleton builder, Dewaterability, Extracellular polymeric substances

Posted Date: May 6th, 2021

DOl: https://doi.org/10.21203/rs.3.rs-341579/v1

License: (c) (i) This work is licensed under a Creative Commons Attribution 4.0 International License. Read Full License

Version of Record: A version of this preprint was published at Environmental Science and Pollution Research on October 7th, 2021. See the published version at https://doi.org/10.1007/s11356-021-16404- 

$2 \mathrm{Fe}^{2+} /$ persulfate and skeleton builder conditioner for waste

\section{activated sludge dewatering}

5 Xiaoran $\mathrm{Li}^{1}$, Yafei Shi ${ }^{*}{ }^{1,2}$, Xi Zhou ${ }^{1}, \mathrm{Lu} \mathrm{Wang}^{1}$, Huiqin Zhang ${ }^{1}, \mathrm{Kewu} \mathrm{Pi}^{1,2}$, Andrea

$6 \quad$ R. Gerson ${ }^{3}$, Defu Liu ${ }^{1,2}$

$8{ }^{1}$ School of Civil Engineering, Architecture and Environment, Hubei University of

9 Technology, Wuhan, Hubei 430068, China

${ }^{2}$ Hubei Key Laboratory of Ecological Restoration for River-Lakes and Algal

11 Utilization, Wuhan, Hubei, 430068, China

${ }^{3}$ Blue Minerals Consultancy, Wattle Grove, Tasmania, 7109, Australia

$13{ }^{*}$ Corresponding at. E-mail: yfshi@hbut.edu.cn (Yafei Shi) 


\section{Abstract}

Sludge conditioning is important for improved dewatering, with the sludge characteristics impacting the effect of conditioning. A composite conditioner, $\mathrm{Fe}^{2+}$ activated sodium persulfate ( $\left.\mathrm{Fe}^{2+} / \mathrm{SPS}\right)$ combined with phosphogypsum (PG), was used to examine its impact on sludges with different organic content or different solid content. Response surface optimization analysis shows that when the best conditioning is achieved, the reduction of the specific resistance to filtration is not sensitive to organic matter content, but the dewatering performance of the sludge is greatly affected by the solid content. The oxidation role of $\mathrm{Fe}^{2+} / \mathrm{SPS}$ and the skeleton builder role of $\mathrm{PG}$, together, affect the conditioning, with oxidation playing a major role in conditioning, especially for greater organic matter content. The organic content also affects the effectiveness of the skeleton builder more than the solid content. Changes in PG significantly impacts the optimal molar ratio and dosage of $\mathrm{Fe}^{2+} / \mathrm{SPS}$. Sludge with greater solid content requires greater $\mathrm{Fe}^{2+} / \mathrm{SPS}$ dosage to provide stronger oxidation to destroy flocs. The composite conditioning decreases the content of extracellular polymeric substances and proteins/polysaccharides with the decrease in protein content being significantly greater than that of polysaccharides, these decreases improve the sludge dewaterability.

Keywords: Sewage sludge; Conditioning; Persulfate oxidation; Skeleton builder; Dewaterability; Extracellular polymeric substances 


$\begin{array}{ll}\text { CS } & \text { Conditioned sludge } \\ \text { DS } & \text { Dry solid } \\ \text { EPS } & \text { Extracellular polymeric substances } \\ \text { LB-EPS } & \text { Loosely bound-EPS } \\ \text { OMC } & \text { Organic content } \\ \text { PG } & \text { Phosphogypsum } \\ \text { PN } & \text { Protein } \\ \text { PS } & \text { Polysaccharides } \\ \text { RS } & \text { Raw sludge } \\ \text { RSM } & \text { Response surface methodology } \\ \text { SC } & \text { Solid content } \\ \text { SF } & \text { Fe }{ }^{2+} / \mathrm{S}_{2} \mathrm{O}_{8}{ }^{2-} \\ \text { SPS } & \text { Sodium persulfate } \\ \text { SRF } & \text { Specific resistance to filtration } \\ \text { TB-EPS } & \text { Tightly bound-EPS } \\ \text { WC } & \text { Water content }\end{array}$




\section{Introduction}

With the growth of urban populations and the continuous improvement of municipal service facilities and sewage treatment technology, the amount of sewage sludge produced by urban sewage treatment plants is increasing. The effectiveness of its treatment has become an important issue. Treatment and disposal costs account for about $40-50 \%$ of the total operating costs of sewage treatment plants (Xiao et al. 2017). Sewage treatment plants often use conditioning before mechanical dewatering for improved sludge dewatering performance (Cao et al. 2021, Wu et al. 2020).

Commonly used sludge conditioning methods are physical conditioning (Carrasco and Gao 2019, Liu et al. 2019, Mobaraki et al. 2018, Ramachandra and Devatha 2020), chemical conditioning (Ge et al. 2019, Hu et al. 2020, Wang et al. 2018, Wang et al. 2019, Yu et al. 2019) and biological conditioning (Chen et al. 2015, Huo et al. 2014, Liu et al. 2016a). Advanced oxidation technology has become a subject of research interest in the area of chemical conditioning due to the advantages of low secondary pollution and fast reaction times (Bian et al. 2021, Chen et al. 2021, Chen et al. 2020, Kim et al. 2016, Ni et al. 2019, Wei et al. 2020, Zhang et al. 2020b). Our previous research used $\mathrm{Fe}^{2+}$ activated sodium persulfate (SPS) combined with phosphogypsum (PG) for sludge conditioning and found both oxidation and skeleton building effects (Shi et al. 2015). $\mathrm{Fe}^{2+} / \mathrm{SPS}$ oxidation promoted the rapid generation of column-shaped dihydrated phases of $\mathrm{CaSO}_{4} \cdot 2 \mathrm{H}_{2} \mathrm{O}$ from the hemihydrate phases of $\mathrm{CaSO}_{4} \cdot 0.5 \mathrm{H}_{2} \mathrm{O}$. Newly generated column-shaped dihydrate phases acted as skeleton builders, thus improving sludge dewatering. There are many studies focus on the chemical conditioner's selection (Badalians Gholikandi et al. 2018, Xiao et al. 2020, Zhang et al. 2019), optimization (He et al. 2020, He et al. 2015, Rumky et al. 2018, Yu et al. 2017) and combination (Guo and Zhou 2020, Guo et al. 2020, Wu et al. 2019a, Wu et al. 2019b), but it is uncertain whether differences in sludge characteristics causes uncertainty in the choice and effectiveness of conditioners.

The characteristics of sludge and the interactions of sludge agents are closely related to the performance of agents (Zhang et al. 2020a). High water content and high 
organic matter content are the main factors limiting dewatering of the sludge. Currently, the dosage of sludge conditioning is generally based on sludge volume (per liter) (Lu et al. 2003), sludge dry solids (per g of DS) (Guo et al. 2019a, Liang et al. 2015, Shi et al. 2015) or sludge organic matter content (per g volatile suspended-solid) (Zhen et al. 2012). However, due to the differences in properties of sludge, such as moisture content and organic matter, the same dosage will result in highly variable conditioning effects. Our previous research showed that among the many characteristic indicators of sludge, initial solid content and volatile suspended-solid/total suspended solid were sludge characteristics that most affect the solid content of the dewatered cake with $\mathrm{Fe}^{2+} / \mathrm{S}_{2} \mathrm{O}_{8}{ }^{2-}$ -phosphogypsum composite ( $\mathrm{Fe}^{2+} / \mathrm{SPS}-\mathrm{PG}$ ) conditioning (Shi et al. 2016). Yu et al. (2017) used sludge dry solid and volatile solid content to optimize the dosage of different organic sludges by Fenton oxidation. The results showed that optimization of oxidation reagent based on volatile solid content is more plausible than that of based on dry solid content for different sewage sludge with different organic matter contents.

Although different method or evaluation have been investigated in sludge conditioning, careful analysis is required to determine how the variability of solid content and volatile suspended-solid/total suspended solid affects optimal dose, oxidative effects and skeleton structure. Therefore, the main aim of this study is to investigate the impact of different solid content and organic content on $\mathrm{Fe}^{2+} / \mathrm{SPS}-\mathrm{PG}$ composite conditioning of different sludge types. The specific tasks were to: (i) optimize the dosage $\mathrm{Fe}^{2+} / \mathrm{SPS}-\mathrm{PG}$ and determine the dewaterability of each sludge with different solid content and organic content in laboratory optimization experiments. (ii) identify the effectiveness between $\mathrm{Fe}^{2+} / \mathrm{SPS}$ oxidation and PG skeleton builder, so as to determine the use of compound conditioning strategies according to the determined optimal conditions.

\section{Materials and methods}

\subsection{Materials}

A waste sewage sludge sample was taken from the Shahu wastewater treatment 
94 plant, Wuhan, China. Large debris such as stones, branches and leaves were removed using a $2 \mathrm{~mm}$ sieve. To obtain a good reproducibility, all tests were completed within 3 days and the sludge was stored at $4{ }^{\circ} \mathrm{C}$. The sludge was naturally settled to remove supernatant or was supplemented with a quantitative amount of distilled water to obtain sludge samples with the same organic content but different solid content (denoted as SC). Similarly, if the water content of the sampled sludge samples is kept the same, sludge samples with the same solid content but different organic content (denoted as OMC) can be obtained. The basic characteristics of the sludge are shown in Table 1. Sodium persulfate (SPS) $\left(\mathrm{Na}_{2} \mathrm{~S}_{2} \mathrm{O}_{8}\right.$, purity $\left.>99.9 \mathrm{wt} \%\right)$ and ferrous sulfate $\left(\mathrm{FeSO}_{4} \cdot 7 \mathrm{H}_{2} \mathrm{O}\right.$, purity $\left.>99.9 \mathrm{wt} \%\right)$ were analytical reagents (Sinopharm company, Shanghai, China). The dissolved $\mathrm{Fe}^{2+}$ solutions were prepared from $\mathrm{FeSO}_{4} \cdot 7 \mathrm{H}_{2} \mathrm{O}$ in ultrapure water. Thermally-pretreated PG, used as a skeleton builder, was sieved using a mesh size of $0.08 \mathrm{~mm}$ after heating at $150{ }^{\circ} \mathrm{C}$ for $2 \mathrm{~h}$ (Shi et al. 2015).

\subsection{Conditioning procedure}

Sludge conditioning experiments were conducted using a programmable jar test apparatus. Sludge $(300 \mathrm{~mL})$ was placed in a $500 \mathrm{~mL}$ beaker. SPS was added (mg per g of DS) and stirred at $300 \mathrm{rpm}$ for $15 \mathrm{~min}$. $\mathrm{Fe}^{2+}$ solution was fed into the slurry and stirred at $150 \mathrm{rpm}$ for $15 \mathrm{~min}$ (1/3 of the total amount was added every $5 \mathrm{~min}$ ). Finally, PG was added and with stirring for $5 \mathrm{~min}$ at $150 \mathrm{rpm}$ to complete the conditioning process. Each experiment was carried out three times. A blank control experiment, using raw sludge (RS), was carried out without conditioner.

\subsection{Specific resistance to filtration}

After conditioning, $100 \mathrm{~mL}$ of the conditioned sludge was poured into a $9 \mathrm{~cm}$ standard Buchner funnel fitted with pre-wetted qualitative filter paper. A constant vacuum pressure of $80 \mathrm{kPa}$ was then applied until no further filtrate was obtained. Both the filtrate volume and the sludge cake weight were recorded. The moisture content of the filter cake was then determined. Whereafter, the specific resistance to filterability (SRF) was calculated according to Eq. 1. 
$P$ is the filtration pressure $(\mathrm{Pa}) ; A$ is the area of the filter cake $\left(\mathrm{m}^{2}\right) ; b\left(\mathrm{~s} / \mathrm{m}^{6}\right)$ is the slope determined from the $t / V$ versus $V$ plot, ( $V$ is the volume of filtrate, $\mathrm{m}^{3}$; and $t$ is the filtration time (s)); $\mu$ is the viscosity of the filtrate $(\mathrm{Pa} \cdot \mathrm{s}) ; \omega$ is the sludge solids concentration $\left(\mathrm{kg} / \mathrm{m}^{3}\right)$.

The reduction of SRF is expressed by Eq. 2.

$$
\text { Reduction of } \mathrm{SRF}=\left(\left(S R F_{r}-S R F_{c}\right) /_{S R F_{r}}\right) \times 100 \%
$$

$S R F_{r}$ is the specific resistance to filterability of $\mathrm{RS}(\mathrm{m} / \mathrm{kg})$ and $S R F_{c}$ is average value of the specific resistance to filterability of the conditioned sludge (CS) (m/kg).

The DS content was measured by evaporation at $105{ }^{\circ} \mathrm{C}$ for $24 \mathrm{~h}$. Organic matter content was determined using muffle furnace by ignition at $600{ }^{\circ} \mathrm{C}$ for $3 \mathrm{~h}$. Sludge moisture content, $\mathrm{pH}$ and other indicators were determined by the methods defined in "CJ/T221-2005 Sludge Inspection Method for Municipal Sewage Treatment Plant" (China standard for municipal sludge analysis).

\subsection{Response surface methodology}

A central composite design based on response surface methodology (RSM) was employed to optimize dosages of SPS, $\mathrm{Fe}^{2+}$ and PG. Ranges and levels of these three constituents were defined based on the preliminary tests shown in Table 2. The SRF and water content of the filter cakes were considered as the responses. Eighteen runs were required for one set of experiments for each sludge type. The experimental schemes and results are shown in Table S1. For optimization, we firstly analyzed each response to establish the appropriate model using Design Expert 8 software, then searched for a combination of factor levels that simultaneously satisfy the requirements. The goal of the numerical optimization was to obtain the dosage of $\mathrm{Fe}^{2+}$ and SPS, setting the target value of the constraint PG dosage and SRF within the range of experimental design.

\subsection{Net sludge solids yield}

The net sludge solids yield $\left(Y_{N}\right)$ refers to the solid content of the sludge filtered per 
unit time and unit filtration area. Generally, when large amounts of skeleton builders are added to the sludge, the solid concentration of the sludge changes, so the filterability is expressed as the theoretical yield $Y$ (Eq. 3). In order to express $Y_{N}$, a correction factor $f$ is introduced as per Eq. 4 (Benítez et al. 1994).

$$
\begin{aligned}
Y & =(2 P \omega / \mu t S R F)^{1 / 2} \\
Y_{N} & =f(2 P \omega / \mu t S R F)^{1 / 2}
\end{aligned}
$$

The dose of skeleton builder added (SOL) is defined by Eq. 5 .

$$
S O L=\left(\frac{\text { mass of skeleton builder added }}{\text { mass of dry solids in the original sludge }}\right) \times 100 \%
$$

Then, $f$ can be expressed as Eq. 6 :

$$
f=1 /(1+(S O L / 100 \%))
$$

Finally, the effect of the skeleton builder on the filterability of sludge, $\eta_{S O L}$, is defined by Eq. 7.

$$
\eta_{S O L}=\left(\left(\left(Y_{N}\right)_{o p t}-\left(Y_{N}\right)_{0}\right) /\left(Y_{N}\right)_{o p t}\right) \times 100 \%
$$

$\left(Y_{N}\right)_{\text {opt }}$ is the net yield when conditioning with optimal dosage of $\mathrm{Fe}^{2+} / \mathrm{SPS}-\mathrm{PG} ;\left(Y_{N}\right)_{0}$ is the net yield when conditioning only with $\mathrm{Fe}^{2+} / \mathrm{SPS}$.

\subsection{Extracellular polymeric substances (EPS) extraction and analysis}

A modified heat extraction method was used to extract the slime (S-EPS), the loosely bound EPS (LB-EPS) and tightly bound EPS (TB-EPS) (Li \&Yang 2007). Sludge $(50 \mathrm{~mL}$ ) was centrifuged at $4,000 \mathrm{~g}$ for $5 \mathrm{~min}$. The supernatant was collected for analysis of S-EPS. The sludge pellet in the tube was then diluted to its original volume of $50 \mathrm{~mL}$ with $0.05 \mathrm{wt} \%$ sodium chloride $(\mathrm{NaCl})$ solution pre-heated to $70{ }^{\circ} \mathrm{C}$. The sludge sample was then immediately sheared using a vortex mixer for $1 \mathrm{~min}$, followed by centrifugation at 4,000 $\mathrm{g}$ for $10 \mathrm{~min}$. The supernatant was then collected for analysis of the LB-EPS. The residual sludge pellet was re-suspended in $0.05 \mathrm{wt} \%$ $\mathrm{NaCl}$ solution to its original volume of $50 \mathrm{~mL}$ and heated to $60{ }^{\circ} \mathrm{C}$ in a water bath for $30 \mathrm{~min}$, then centrifuged for $15 \mathrm{~min}$, and the supernatant was collected for analysis of 
$201 \quad \mathrm{Y}_{\mathrm{S} 3}$

$202=0.15-0.14 \mathrm{~A}-0.11 \mathrm{~B}-0.082 \mathrm{C}-0.052 \mathrm{AB}-\left(6.30 \times 10^{-3} \mathrm{AC}\right)+0.030 \mathrm{BC}$

$203+0.092 \mathrm{~A}^{2}+0.15 \mathrm{~B}^{2}$

$204+0.014 C^{2}$

A quadric surface model was established for the SRF value, water content (WC), and organic matter content of the filter cakes of the different sludges. Variance analysis and regression coefficient significance tests were performed, as shown in Table S2. It can be seen that the models (Table S2) are all significant and have good fit, indicating that the models are appropriate, and predictive analysis can be performed. For example, the quadratic regression model equation for the SRFs of the sludges were established to be:

$$
\begin{aligned}
\mathrm{Y}_{\mathrm{S} 1}=0.11- & 0.058 \mathrm{~A}-0.18 \mathrm{~B}-0.071 \mathrm{C}-0.03 \mathrm{AB}+\left(2.87 \times 10^{-3} \mathrm{AC}\right)+0.044 \mathrm{BC} \\
& +0.049 \mathrm{~A}^{2}+0.19 \mathrm{~B}^{2} \\
& +\left(7.99 \times 10^{-3} \mathrm{C}^{2}\right) \\
& =0.998
\end{aligned}
$$




$$
\begin{aligned}
\mathrm{Y}_{\mathrm{S} 4}=0.14- & 0.12 \mathrm{~A}-0.087 \mathrm{~B}-0.095 \mathrm{C}+\left(9.12 \times 10^{-4} \mathrm{AB}\right)+0.024 \mathrm{AC}+0.021 \mathrm{BC} \\
& +0.11 \mathrm{~A}^{2}+0.11 \mathrm{~B}^{2} \\
& +\left(2.54 \times 10^{-3} \mathrm{C}^{2}\right) \\
& =0.996
\end{aligned}
$$

$\mathrm{Y}_{\mathrm{S} 5}$

$=0.1-\left(9.97 \times 10^{-3} \mathrm{~A}\right)+\left(3.06 \times 10^{-3} \mathrm{~B}\right)-0.06 \mathrm{C}-0.026 \mathrm{AB}-\left(7.87 \times 10^{-3} \mathrm{AC}\right)$

$+\left(5.68 \times 10^{-3} \mathrm{BC}\right)+0.014 \mathrm{~A}^{2}+\left(3.81 \times 10^{-3} \mathrm{~B}^{2}\right)$

$+0.02 \mathrm{C}^{2}$

$=0.990$

$$
\begin{aligned}
& Y_{S 6} \\
& =0.067+\left(1.70 \times 10^{-3} A\right)-0.023 B-0.057 C-\left(5.75 \times 10^{-3} A B\right)-0.015 A C
\end{aligned}
$$$$
+0.036 \mathrm{BC}+0.018 \mathrm{~A}^{2}+\left(6.69 \times 10^{-3} \mathrm{~B}^{2}\right)
$$$$
+0.034 \mathrm{C}^{2}
$$$$
=0.995
$$

$$
\mathrm{Y}_{\mathrm{S} 7}
$$$$
=0.11-0.018 \mathrm{~A}-0.086 \mathrm{~B}-0.075 \mathrm{C}-\left(1.05 \times 10^{-3} \mathrm{AB}\right)-0.016 \mathrm{AC}+0.041 \mathrm{BC}
$$$$
+0.012 \mathrm{~A}^{2}+0.091 \mathrm{~B}^{2}
$$

$-0.026 \mathrm{C}^{2}$

$=0.997$

$$
\begin{aligned}
& Y_{S 8} \\
& =0.14-0.013 \mathrm{~A}-0.14 \mathrm{~B}-0.048 \mathrm{C}-\left(2.91 \times 10^{-4} \mathrm{AB}\right)+\left(9.10 \times 10^{-5} \mathrm{AC}\right)
\end{aligned}
$$$$
+0.02 \mathrm{BC}+0.021 \mathrm{~A}^{2}+0.13 \mathrm{~B}^{2}
$$$$
-0.036 \mathrm{C}^{2}
$$

$$
=0.997
$$

Where: A, B, C stand for SPS, $\mathrm{Fe}^{2+}$ and PG, Respectively.

\subsection{Influence of organic matter content on sludge dewaterability}

To analyze the difference in the dewatering performance due to $\mathrm{OMC}$, the lowest SRF value was set as the goal, and the dosage of various OMC agents was optimized by RSM. It can be seen from Table 3 that when the best conditioning is achieved (that is, the lowest SRF value), the dosage is variable, but the change of the reduction of SRF is stable above $98 \%$. This shows that $\mathrm{Fe}^{2+} / \mathrm{SPS}-\mathrm{PG}$ has a stable conditioning effect on different OMC. In addition, it was found that with the increase of organic matter content, the $\left(\mathrm{Y}_{\mathrm{N}}\right)_{\mathrm{opt}}$ value increased and then decreased, with the trend for SRF reversed. When 
the organic content is $39.3 \%,\left(\mathrm{Y}_{\mathrm{N}}\right)_{\mathrm{opt}}$ is 1.919 , and the sludge filtration performance is the best.

From the perspective of the effectiveness of the skeleton builder, although the $\eta_{\text {SOL }}$ value is variable $(0.078-0.187)$, all sludges reached a stable maximum reduction of SRF. This indicates that the oxidation effect of activated persulfate and the skeleton effect of PG can work together to achieve the best conditioning effect.

The nature of the raw sludge determines the SRF of the conditioned sludge. It can be seen from Fig. 1 that with increasing organic matter content, the SRF values of the raw sludge and conditioned sludge decrease and then increase, but the final reduction of SRF is basically unchanged (Table 3). For the OMC with the same moisture content, on the one hand, adding the same agent does not change the sludge moisture content of the conditioning system (e.g., $\mathrm{S}_{1}-\mathrm{S}_{4}, \mathrm{~S}_{6}$ ). The $\mathrm{Fe}^{2+}$ :SPS molar ratio for optimal conditioning of the different sludges is similar and the reaction produces a similar number of $\mathrm{SO}_{4} \bullet^{-}$, so $\mathrm{SO}_{4} \bullet^{-}$"effective reaction concentration" is equivalent (that is, the relative concentration or dose that causes the equivalent oxidation reaction), free radicals destroy the extracellular polymeric substance within the sludge and release intracellular water (Zhen et al. 2012), resulting in the same reduction of SRF of sludge with organic content of $34.6-43.8 \%$. This shows that the $\mathrm{Fe}^{2+} / \mathrm{SPS}-\mathrm{PG}$ conditioning system has a stable conditioning effect on different OMC and is suitable for sludge conditioning with low and high organic content.

When the sludge organic matter is the same, the SRF value decreases with the increasing PG dosage (Fig. 2). When the PG dosage exceeds $100 \mathrm{mg} / \mathrm{g}$ DS, the change trend of the reduction of SRF of different OMC is stable, which is consistent with the conclusion that $\mathrm{Fe}^{2+} / \mathrm{SPS}-\mathrm{PG}$ conditioning has a wide application range for OMC. However, with increasing organic matter content, the SRF value decreases and then increases. The trend of SRF values of the conditioned sludge is affected by the nature of raw sludge. A higher organic content (organic content $>39.3 \%$ ) usually contains greater EPS content, which is not conducive to improving the separation of water from the filter cake (Wang et al. 2020). When the molar ratio of $\mathrm{Fe}^{2+}: \mathrm{SPS}$ is 0.5 , the SRF 
value for the different OMC is the greatest, and the reduction of SRF varies significantly. This may be because the $\mathrm{Fe}^{2+}$ dosage is insufficient and the SPS cannot be activated sufficiently to produce sufficient free radicals, resulting in the sub-optimal destruction of organic components in the sludge.

When the sludge organic matter is the same, the $\mathrm{Fe}^{2+}$ :SPS molar ratio is basically unchanged with increasing PG dosage, the $\eta_{\text {SOL }}$ value gradually decreases, and the $\mathrm{Y}_{\mathrm{N}}$ value gradually increases (Fig. 3). This indicates that the oxidation of $\mathrm{S}_{2} \mathrm{O}_{8}{ }^{2-}$ plays a major role in the conditioning system, and the role of $\mathrm{PG}$ as a skeleton builder is masked by the oxidation of sulfate radicals. The same PG dosage results in variable changes in $\eta_{\text {SOL }}$ and $Y_{N}$ values for different $\mathrm{OMC}$, indicating that PG has different effects on the conditioning of different OMC. With PG dosage of $100 \mathrm{mg} / \mathrm{g}$ DS, the $\eta_{\text {SOL }}$ value is larger than at greater dosage, and the $\eta_{\text {SOL }}$ value of the sludge with organic matter of $34.6 \%$ is the largest, indicating that the low dosage of PG has the greater impact on sludge with low organic matter content. On PG dosage greater than $100 \mathrm{mg} / \mathrm{g}$ DS, the $\eta_{\text {SOL }}$ value for different OMC does not change appreciably, which shows that advanced oxidation plays a major role in the conditioning system, and the excessive addition of PG leads to a little impact on the sludge filtration performance.

\subsection{Influence of solid content on sludge dewaterability}

The RSM optimization experiment results of four different solids contents (SC, $\mathrm{S}_{5}-\mathrm{S}_{8}$ ) conditioning show that the dosages of agents corresponding to the minimum SRF value of different SCs are the same, but the SRF value is different (Table 4). This shows that the SC affects the sludge dewatering performance significantly.

As the SC increases, the $\left(\mathrm{Y}_{\mathrm{N}}\right)_{\text {opt }}$ value increases and then decreases, with the trend of the SRF value being the reverse. These two indicators show that for the SC of 3.8 $w t \%$, the best conditioning is achieved with the maximum $\eta_{\text {SOL }}$ value of 0.147 , indicating that for different SC, the $300 \mathrm{mg} / \mathrm{g}$ DS PG dosage has the greatest impact on the filterability of the sludge. 
As the solid content increases, the SRF value first decreases and then increases, while the trend of the reduction of SRF is the reverse (Fig. 4). For sludge with high SC, a portion of the PG forms a skeleton providing water filtration channels, but a greater dosage of PG causes to much skeleton to be built. The viscosity of the system increases, and the sludge filtration performance deteriorates.

The optimum dosage ratio of $\mathrm{Fe}^{2+}$ and SPS for different SCs with different PG doses varies, as does the extent of conditioning (Fig. 5). This shows that the dewatering performance of sludge is closely related to SC and reasonable dosage ratio. When the $\mathrm{Fe}^{2+}:$ SPS molar ratio is 0.5 , the SRF value for different SC is relatively large, and the SRF value of sludge with low SC is the largest. Low SC means more water content, resulting in the agent concentration being "diluted", thereby reducing the effectiveness of conditioning. If the $\mathrm{Fe}^{2+}$ :SPS molar ratio is too small, so that the $\mathrm{Fe}^{2+}$ dosage is insufficient, the effective decomposition of SPS is limited as is the production of $\mathrm{SO}_{4}{ }^{-}$, weakening the intensity of oxidation of the sludges (Luo et al. 2018, Ni et al. 2019). With PG dosage of $100 \mathrm{mg} / \mathrm{g}$ DS and the $\mathrm{Fe}^{2+}$ :SPS molar ratio is greater than 0.5 , the SRF value gradually decreases with SC decreases, and the oxidation efficiency of $\mathrm{SO}_{4} \bullet^{-}$ on sludge increases, thereby improving the filtration performance.

To analyse the dynamic relationship between dosage of $\mathrm{PG}$ and $\mathrm{Fe}^{2+}$ :SPS mol ratio with different solid contents. The dosage of $\mathrm{Fe}^{2+}$ and SPS was obtained by numerical optimization, setting the target value of the constraint PG dose and SRF. It can be found that when the SC of sludge is the same, with increasing PG dosage, the $\mathrm{Fe}^{2+}$ :SPS molar ratio shows a downward trend. However, for different SC, it shows different trends. When PG is $100 \mathrm{mg} / \mathrm{g} \mathrm{DS}$, the $\mathrm{Fe}^{2+}$ :SPS molar ratio gradually increases with the increase of SC; when PG is $300 \mathrm{mg} / \mathrm{g} \mathrm{DS}$, the $\mathrm{Fe}^{2+}$ :SPS molar ratio is basically not affected by the SC. This is because when the dosage of PG is small, the sludge with high SC needs a greater $\mathrm{Fe}^{2+}$ :SPS molar ratio to generate more sulfate radicals oxidation to destroy the sludge flocs, and release the bound water to improve the sludge dewatering performance (Zhen et al. 2019). With the PG dosage of $300 \mathrm{mg} / \mathrm{g}$ DS, the hydration and skeleton builder effects of PG gradually become obvious during the conditioning process, which balances out the oxidation effect, so the effect of the molar 
ratio of $\mathrm{Fe}^{2+}:$ SPS becomes stable.

When the SC of the sludge is the same, with increasing PG dosage, the $\eta_{\text {SOL }}$ value gradually decreases and the $Y_{N}$ value gradually increases. With PG dosage of $100 \mathrm{mg} / \mathrm{g}$ DS, with increasing SC, the $\eta_{\text {SOL }}$ value gradually decreases, but the $Y_{N}$ value remains basically unchanged. This also shows that low dosage of PG has little effect on the conditioning of the sludge with high SC, and oxidation is dominant. Furthermore, it can be seen that the $\eta_{S O L}$ values of the variable OMCs is greater than the $\eta_{S O L}$ values of the variable SCs from (Figs. 3 and 6). This shows that for different OMCs, changes in PG dosage are more likely to cause changes in conditioning effects.

\subsection{Changes of EPS characteristic after sludge conditioning}

The EPS content in the sludge has an important influence on the sludge dewatering performance. The EPS content and composition change are shown in Fig. 7 after conditioning the different SC sludges using the $\mathrm{Fe}^{2+}$ :SPS molar ratio of 0.9 and $\mathrm{PG}$ dosage of $300 \mathrm{mg} / \mathrm{g}$ DS.

The total amount of EPS and the amount of each form (layer) of EPS are significantly smaller in the conditioned sludge than in the RS indicating that $\mathrm{SO}_{4}{ }^{-}$ damages the EPS. The TB-EPS content decreases the most. The oxidative degradation of macromolecular organics into small molecular organics has been found previously to reduce the EPS content and improve filtration performance (Guo et al. 2019b, Liu et al. 2016b, Sari Erkan and Onkal Engin 2020). Further analysis found that the reduction of PN content was significantly greater than that of PS, and the PN/PS of CS was significantly smaller than for RS. This is due to the different chain structure of PN and PS. $\mathrm{SO}_{4} \bullet^{-}$preferentially destroys and degrades hydrophobic organics such as PN in EPS, thus causing the reduction of PN to be significantly greater than that of PS, and PN/PS to decrease, as is consistent with the results of Zhen et al. (2019).

With increasing SC, the reduction of PN and PS content increases and then decreases. PN/PS shows the opposite trend. When the SC is $3.8 \mathrm{wt} \%$, the reduction of the EPS and TB-EPS contents are $85.18 \%$ and $89.09 \%$, respectively. The reduction of 
the PN and PS contents are $90.03 \%$ and $70.05 \%$, respectively and the PN/PS is the smallest at 1.04. This conclusion is the same as Section 3.3, when the sludge SC is 3.8 $\mathrm{wt} \%$ and the $\mathrm{PG}$ dosage is $300 \mathrm{mg} / \mathrm{g} \mathrm{DS}$, the $\mathrm{SRF}$ reach the minimum value. It is shown that the reduction of EPS, TB-EPS and PN/PS is beneficial to the filtration performance of sludge, (Yu et al. 2021) found that retaining the hydrophobic surface EPS is beneficial to sludge dewaterability. The possible reason for this inconsistency with this result is the PG addition effectively forms a stable and rigid structure and promotes the formation of porous dehydration channels.

\section{Conclusions}

$\mathrm{Fe}^{2+} / \mathrm{SPS}-\mathrm{PG}$ conditioning was found to be applicable to both high and low organic matter sludges. Organic matter content was found to have little influence on the dewatering of the sludge. The solid content had a significant influence on the effectiveness of the sludge conditioning. Sludge dewaterability was closely related to the solid content and reasonable dosage ratio of the chemical reagent with a trade-off relationship between the dosage of $\mathrm{PG}$ and the molar ratio of $\mathrm{Fe}^{2+}$ :SPS. Change of PG dosage significantly affected the optimum molar ratio of $\mathrm{Fe}^{2+}:$ SPS. Sludge with greater solid content required larger $\mathrm{Fe}^{2+}$ :SPS to generate more $\mathrm{SO}_{4} \bullet^{--}$to damage the sludge EPS. After chemical conditioning, the content of each type (layer) of EPS of different SC, loosely bound, tightly bound or slime, decreased compared with the RS. The reduction of PN content was significantly higher than that of PS, PN was more easily degraded and oxidized. The decrease of PN/PS changed the hydrophilic/hydrophobic characteristics of EPS and significantly improved sludge dewaterability.

\section{Acknowledgments}

This research was supported by doctoral research of launch fund project of Hubei University of Technology (BSQD2016030), national natural science foundation of China youth fund project (51508214) and the Three Gorges follow-up research project (2017HXXY-05). 
Ethical Approval

$380 \quad$ Not applicable

381

382

Consent to Participate

383 Not applicable

384

385

\section{Consent to Publish}

$386 \quad$ Not applicable

387

388

389

390

391

392

393

394

395

396

397

398

399

400

\section{Authors Contributions}

Xiaoran Li: Data curation, Writing - original draft preparation, Writing - review \& editing.

Yafei Shi: Supervision.

Xi Zhou: Investigation.

Lu Wang: Validation.

Huiqin Zhang: Resources.

Kewu Pi: Conceptualization.

Andrea R. Gerson: Writing - review \& editing.

Defu Liu: Project administration.

All authors read and approved the final manuscript.

\section{Funding}

401

Competing Interests

403 The authors declare that they have no competing interests.

404

405

Availability of data and materials

406 The datasets used and/or analysed during the current study are available from the

407 corresponding author on reasonable request. 


\section{References}

Badalians Gholikandi G, Zakizadeh N, Masihi H (2018) Application of peroxymonosulfate-ozone advanced oxidation process for simultaneous wasteactivated sludge stabilization and dewatering purposes: A comparative study. J Environ Manage 206, 523-531

Benítez J, Rodríguez A, Suárez A (1994) Optimization technique for sewage sludge conditioning with polymer and skeleton builders. Water Res 28, 2067-2073

Bian C, Ge D, Wang G, Dong Y, Li W, Zhu N, Yuan H (2021) Enhancement of waste activated sludge dewaterability by ultrasound-activated persulfate oxidation: Operation condition, sludge properties, and mechanisms. Chemosphere 262, 128385

Cao B, Zhang T, Zhang W, Wang D (2021) Enhanced technology based for sewage sludge deep dewatering: A critical review. Water Res 189, 116650

Carrasco M, Gao W (2019) Sludge particle size and correlation with soluble organic matter and conditioning characteristics after freezing treatments. Water, Air, Soil Pollut 230, 54

Chen K, Liu J, Huang S, Mei M, Chen S, Wang T, Li J (2021) Evaluation of the combined effect of sodium persulfate and thermal hydrolysis on sludge dewatering performance. Environ Sci Pollut R 28, 7586-7597

Chen L, Xiong Q, Li H, Hou H, Zhou M (2020) Enhancement of the sewage sludge dewaterability by using ethanol and Fe(III)-rice husk. Environ Sci Pollut R 27, 8696-8706

Chen Z, Zhang W, Wang D, Ma T, Bai R (2015) Enhancement of activated sludge dewatering performance by combined composite enzymatic lysis and chemical re-flocculation with inorganic coagulants: Kinetics of enzymatic reaction and re-flocculation morphology. Water Res 83, 367-376

DuBois M, Gilles KA, Hamilton JK, Rebers PA, Smith F (1956) Colorimetric Method for Determination of Sugars and Related Substances. Anal Chem 28, 350-356

Frølund B, Palmgren R, Keiding K, Nielsen PH (1996) Extraction of extracellular 
polymers from activated sludge using a cation exchange resin. Water Res 30, $1749-1758$

Ge D, Yuan H, Xiao J, Zhu N (2019) Insight into the enhanced sludge dewaterability by tannic acid conditioning and pH regulation. Sci Total Environ 679, 298-306

Guo J, Jiang S, Pang Y (2019a) Rice straw biochar modified by aluminum chloride enhances the dewatering of the sludge from municipal sewage treatment plant. Sci Total Environ 654, 338-344

Guo J, Zhou Y (2020) Transformation of heavy metals and dewaterability of waste activated sludge during the conditioning by $\mathrm{Fe}^{2+}$-activated peroxymonosulfate oxidation combined with rice straw biochar as skeleton builder. Chemosphere 238,124628

Guo S, Liang H, Bai L, Qu F, Ding A, Ji B, Wang X, Li G (2019b) Synergistic effects of wheat straw powder and persulfate/Fe(II) on enhancing sludge dewaterability. Chemosphere 215, 333-341

Guo Z, Ma L, Dai Q, Ao R, Liu H, Yang J (2020) Combined application of modified corn-core powder and sludge-based biochar for sewage sludge pretreatment: Dewatering performance and dissipative particle dynamics simulation. Environ Pollut 265, 115095

He D-Q, Wang L-F, Jiang H, Yu H-Q (2015) A Fenton-like process for the enhanced activated sludge dewatering. Chem Eng J 272, 128-134

He D-Q, Chen J-Y, Bao B, Pan X-L, Li J, Qian C, Yu H-Q (2020) Optimizing sludge dewatering with a combined conditioner of Fenton's reagent and cationic surfactant. J Environ Sci 88, 21-30

Hu J, Li Z, Zhang A, Mao S, Tao W (2020) Using a strong chemical oxidant, potassium ferrate $\left(\mathrm{K}_{2} \mathrm{FeO}_{4}\right)$, in waste activated sludge treatment: A review. Environ Res 188,109764

Huo M, Zheng G, Zhou L (2014) Enhancement of the dewaterability of sludge during bioleaching mainly controlled by microbial quantity change and the decrease of slime extracellular polymeric substances content. Bioresour Technol 168, 190197 
Kim MS, Lee K-M, Kim H-E, Lee H-J, Lee C, Lee C (2016) Disintegration of waste activated sludge by thermally-activated persulfates for enhanced dewaterability. Environ Sci Technol 50, 7106-7115

Li XY, Yang SF (2007) Influence of loosely bound extracellular polymeric substances (EPS) on the flocculation, sedimentation and dewaterability of activated sludge. Water Res 41, 1022-1030

Liang J, Huang S, Dai Y, Li L, Sun S (2015) Dewaterability of five sewage sludges in Guangzhou conditioned with Fenton's reagent/lime and pilot-scale experiments using ultrahigh pressure filtration system. Water Res 84, 243-254

Liu H, Yang S, Shi J, Xu X, Liu H, Fu B (2016a) Towards understanding the dewatering mechanism of sewage sludge improved by bioleaching processing. Sep Purif Technol 165, 53-59

Liu J, Yang Q, Wang D, Li X, Zhong Y, Li X, Deng Y, Wang L, Yi K, Zeng G (2016b) Enhanced dewaterability of waste activated sludge by $\mathrm{Fe}(\mathrm{II})$-activated peroxymonosulfate oxidation. Bioresour Technol 206, 134-140

Liu R, Yu X, Yu P, Guo X, Zhang B, Xiao B (2019) New insights into the effect of thermal treatment on sludge dewaterability. Sci Total Environ 656, 1082-1090

Lu M-C, Lin C-J, Liao C-H, Huang R-Y, Ting W-P (2003) Dewatering of activated sludge by Fenton's reagent. Adv Environ Res 7, 667-670

Luo J, Zhang Q, Wu L, Feng Q, Fang F, Xue Z, Li C, Cao J (2018) Improving anaerobic fermentation of waste activated sludge using iron activated persulfate treatment. Bioresour Technol 268, 68-76

Mobaraki M, Semken RS, Mikkola A, Pyrhönen J (2018) Enhanced sludge dewatering based on the application of high-power ultrasonic vibration. Ultrasonics 84, $438-445$

Ni B-J, Yan X, Sun J, Chen X, Peng L, Wei W, Wang D, Mao S, Dai X, Wang Q (2019) Persulfate and zero valent iron combined conditioning as a sustainable technique for enhancing dewaterability of aerobically digested sludge. Chemosphere 232, 45-53

Ramachandra RH, Devatha CP (2020) Experimental investigation on sludge dewatering 
using granulated blast furnace slag as skeleton material. Environ Sci Pollut R $27,11870-11881$

Rumky J, Ncibi MC, Burgos-Castillo RC, Deb A, Sillanpää M (2018) Optimization of integrated ultrasonic-Fenton system for metal removal and dewatering of anaerobically digested sludge by Box-Behnken design. Sci Total Environ 645, $573-584$

Sari Erkan H, Onkal Engin G (2020) A comparative study of waste activated sludge disintegration by electrochemical pretreatment process combined with hydroxyl and sulfate radical based oxidants. J Environ Chem Eng 8, 103918

Shi Y, Yang J, Yu W, Zhang S, Liang S, Song J, Xu Q, Ye N, He S, Yang C, Hu J (2015) Synergetic conditioning of sewage sludge via $\mathrm{Fe}^{2+} /$ persulfate and skeleton builder: Effect on sludge characteristics and dewaterability. Chem Eng J 270, $572-581$

Shi Y, Yang J, Liang S, Yu W, Hou H (2016) Principal component analysis on sewage sludge characteristics and its implication to dewatering performance with $\mathrm{Fe}^{2+} /$ persulfate-skeleton builder conditioning. Int J Environ Sci Te 13, 22832292

Wang H-F, Hu H, Wang H-J, Zeng RJ (2018) Impact of dosing order of the coagulant and flocculant on sludge dewatering performance during the conditioning process. Sci Total Environ 643, 1065-1073

Wang H-F, Hu H, Wang H-J, Bai Y-N, Shen X-F, Zhang W, Zeng RJ (2020) Comprehensive investigation of the relationship between organic content and waste activated sludge dewaterability. J Hazard Mater 394, 122547

Wang S, Ma C, Zhu Y, Yang Y, Du G, Li J (2019) Deep dewatering process of sludge by chemical conditioning and its potential influence on wastewater treatment plants. Environ Sci Pollut R 26, 33838-33846

Wei L, Xia X, Zhu F, Li Q, Xue M, Li J, Sun B, Jiang J, Zhao Q (2020) Dewatering efficiency of sewage sludge during $\mathrm{Fe}^{2+}$-activated persulfate oxidation: Effect of hydrophobic/hydrophilic properties of sludge EPS. Water Res 181, 115903

Wu B, Dai X, Chai X (2020) Critical review on dewatering of sewage sludge: Influential 
mechanism, conditioning technologies and implications to sludge re-utilizations. Water Res 180, 115912

Wu J, Lu T, Bi J, Yuan H, Chen Y (2019a) A novel sewage sludge biochar and ferrate synergetic conditioning for enhancing sludge dewaterability. Chemosphere 237, 124339

Wu W, Zhou Z, Yang J, Chen G, Yao J, Tu C, Zhao X, Qiu Z, Wu Z (2019b) Insights into conditioning of landfill sludge by $\mathrm{FeCl}_{3}$ and lime. Water Res 160, 167-177

Xiao J, Ge D, Yuan H, Zhu N (2020) Waste activated sludge conditioning in a new $\mathrm{Fe}^{2+} /$ persulfate/tannic acid process: Effectiveness and optimization study to enhance dewaterability. J Environ Chem Eng 8, 103785

Xiao K, Chen Y, Jiang X, Zhou Y (2017) Evaluating filterability of different types of sludge by statistical analysis: The role of key organic compounds in extracellular polymeric substances. Chemosphere 170, 233-241

Yu W, Yang J, Tao S, Shi Y, Yu J, Lv Y, Liang S, Xiao K, Liu B, Hou H (2017) A comparatively optimization of dosages of oxidation agents based on volatile solids and dry solids content in dewatering of sewage sludge. Water Res 126, $342-350$

Yu W, Wen Q, Yang J, Xiao K, Zhu Y, Tao S, Lv Y, Liang S, Fan W, Zhu S, Liu B, Hou $\mathrm{H}$, $\mathrm{Hu} \mathrm{J}$ (2019) Unraveling oxidation behaviors for intracellular and extracellular from different oxidants $\left(\mathrm{HOCl}\right.$ vs. $\left.\mathrm{H}_{2} \mathrm{O}_{2}\right)$ catalyzed by ferrous iron in waste activated sludge dewatering. Water Res 148, 60-69

Yu W, Wen Q, Yang J, Xiao K, Zhu Y, Tao S, Liang S, Hu S, Wu Q, Hou H, Liu B, Hu J (2021) Novel insights into extracellular polymeric substance degradation, hydrophilic/hydrophobic characteristics, and dewaterability of waste activated sludge pretreated by hydroxylamine enhanced Fenton oxidation. ACS ES\&T Engineering 1, 385-392

Zhang G, Shi Q, Li Q, Wang H, Yuan H, Guo W, Lu Y (2020a) Agents for sludge dewatering in fundamental research and applied research: A bibliometric analysis. J Clean Prod 273, 122907

Zhang W, Cheng H, Peng S, Li D, Gao H, Wang D (2020b) Performance and 
mechanisms of wastewater sludge conditioning with slag-based hydrotalcitelike minerals (Ca/Mg/Al-LDH). Water Res 169, 115265

Zhang X, Kang H, Zhang Q, Hao X, Han X, Zhang W, Jiao T (2019) The porous structure effects of skeleton builders in sustainable sludge dewatering process. J Environ Manage 230, 14-20

Zhen G, Lu X, Zhao Y, Chai X, Niu D (2012) Enhanced dewaterability of sewage sludge in the presence of $\mathrm{Fe}(\mathrm{II})$-activated persulfate oxidation. Bioresour Technol 116, 259-265

Zhen G, Tan Y, Wu T, Wang J, Lu X, Zhao Y, Zhu X, Niu J, Xiong J (2019) Strengthened dewaterability of coke-oven plant oily sludge by altering extracellular organics using Fe(II)-activated persulfate oxidation. Sci Total Environ 688, 1155-1161 
Figures

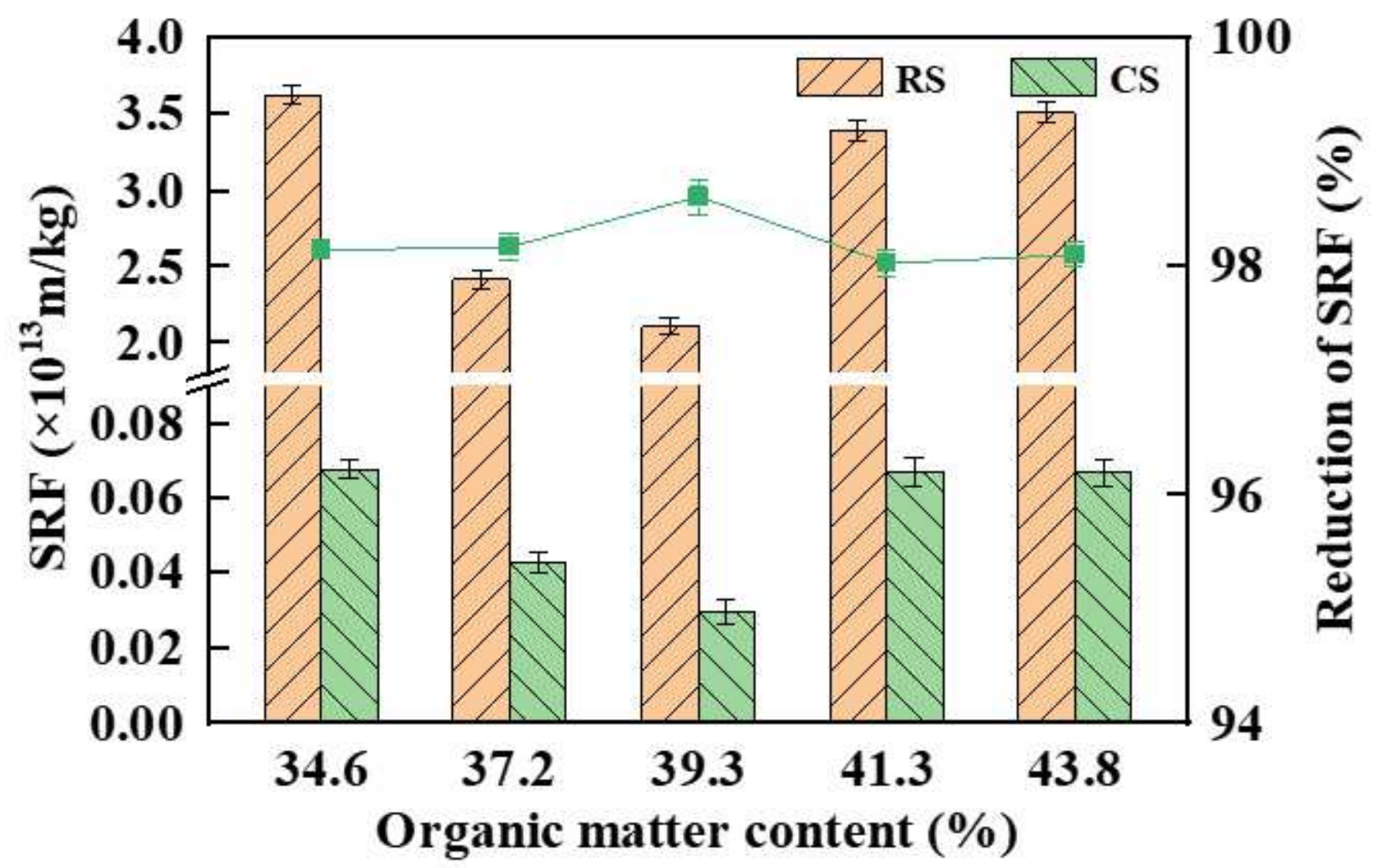

Figure 1

Influence of different organic matter content on the optimal response value SRF. RS $\otimes$ raw sludge; CS $\nabla$ conditioned sludge.

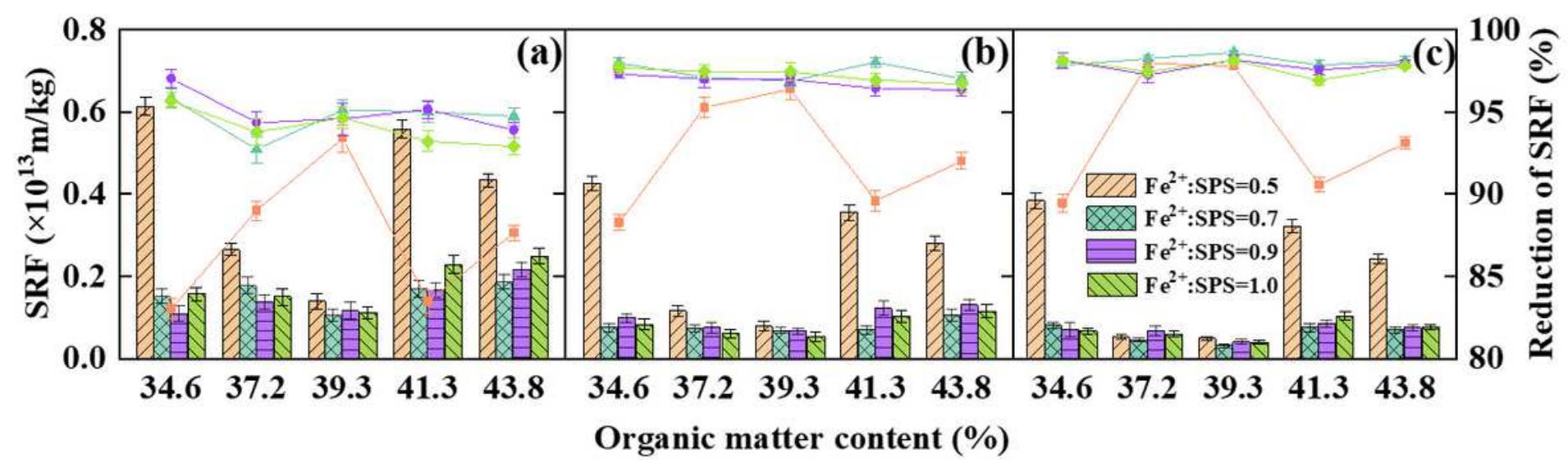

Figure 2 
Influence of different organic matter content on sludge dewaterability. PG dosages (mg/g DS) of (a) 100, (b) 200 and (c) 300. (bars refer to SRF and lines refer to reduction of SRF).

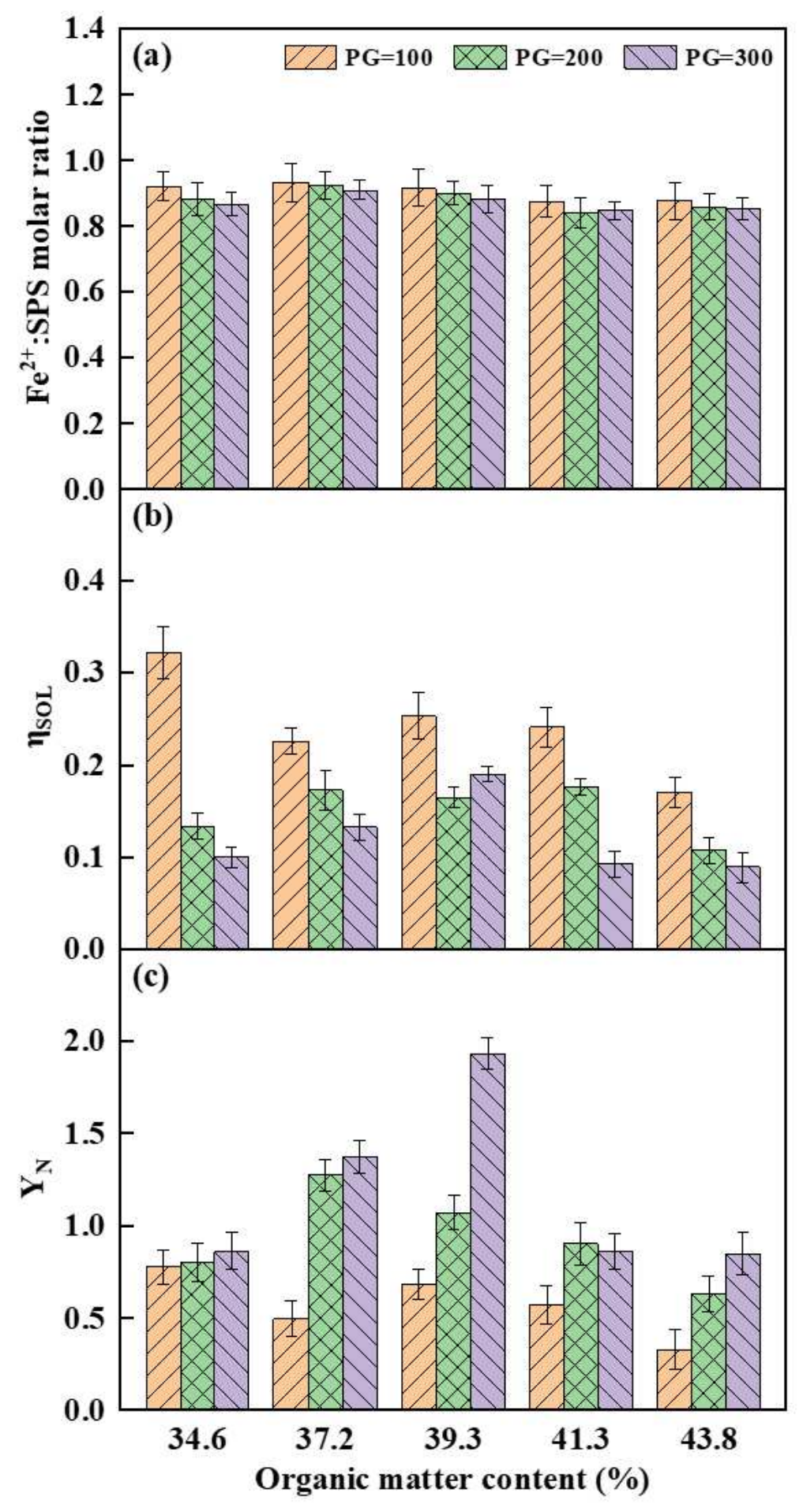

Figure 3

The dynamic relationship between dosage of PG (mg/g DS) and Fe2+:SPS mol ratio with different organic matter contents. 


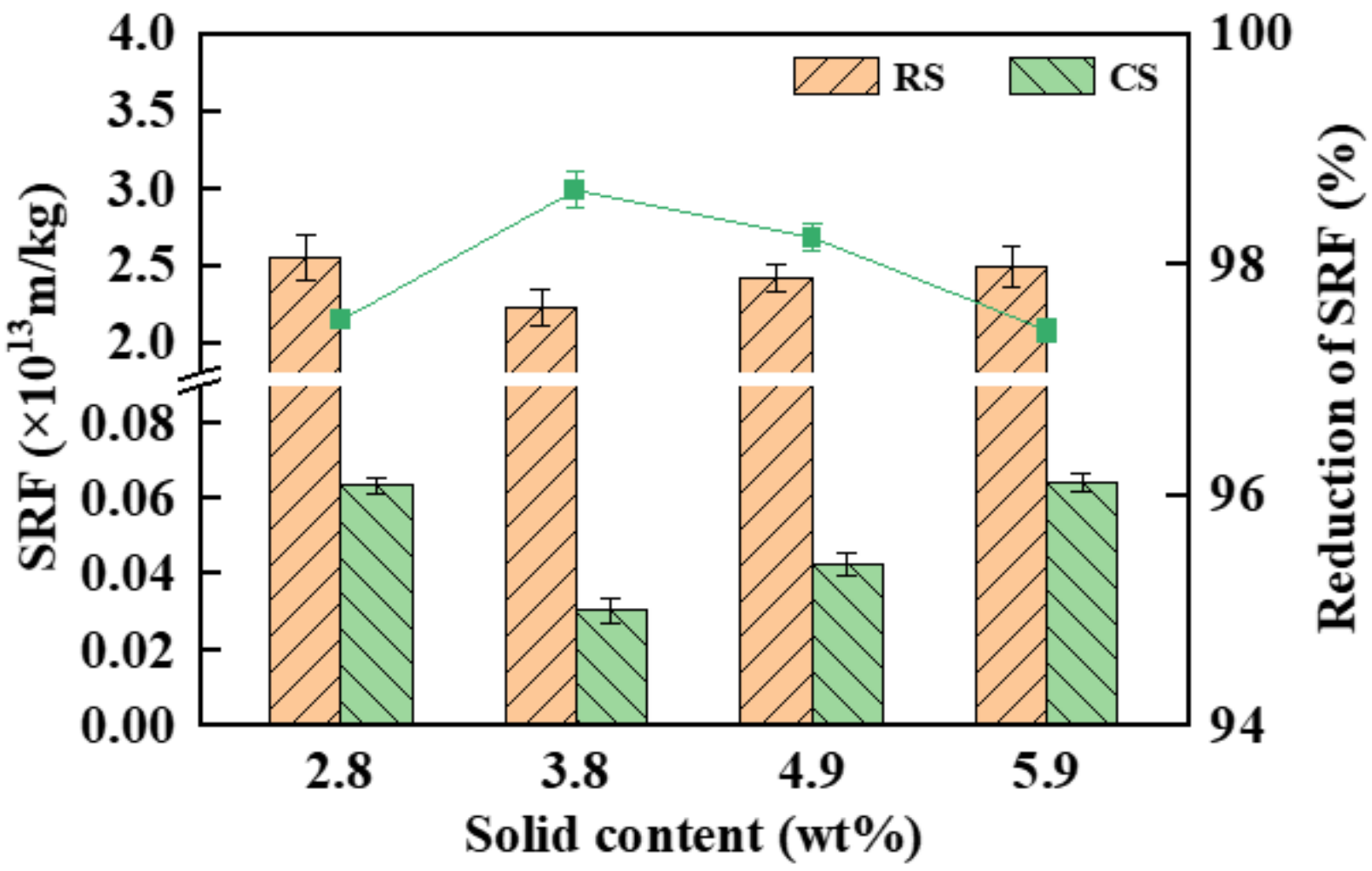

Figure 4

Influence of different solid contents on the optimal response value SRF. RS $\nabla$ raw sludge; CS $\nabla$ conditioned sludge.

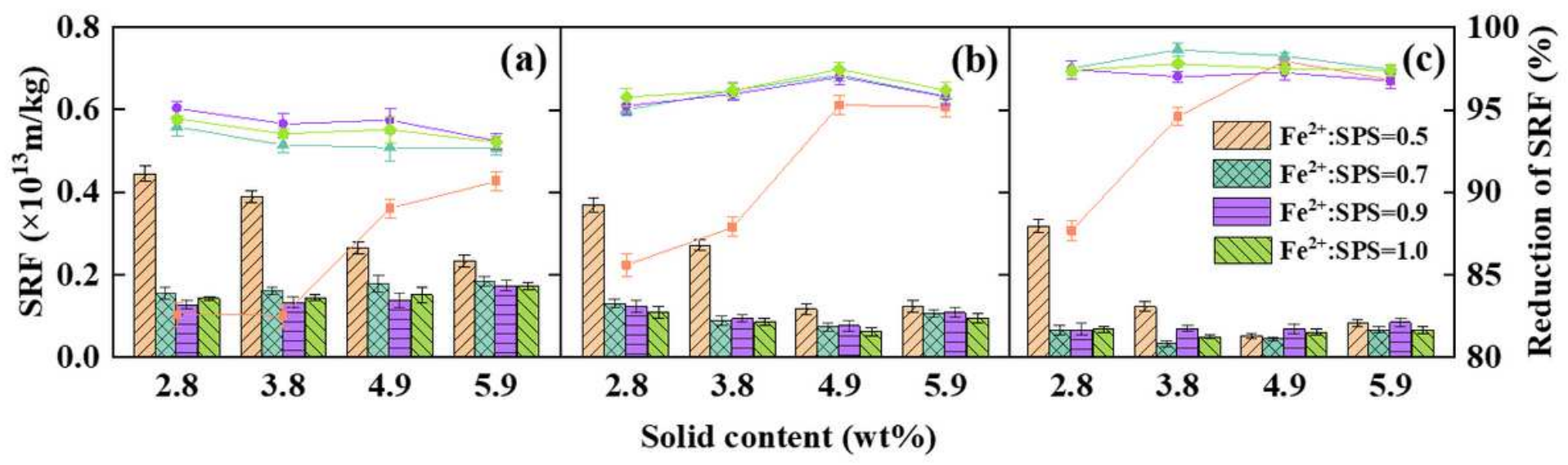

Figure 5

Influence of different solid content on the sludge dewaterability for PG doses (mg/g DS) of (a) 100, (b) 200 and (c) 300. 


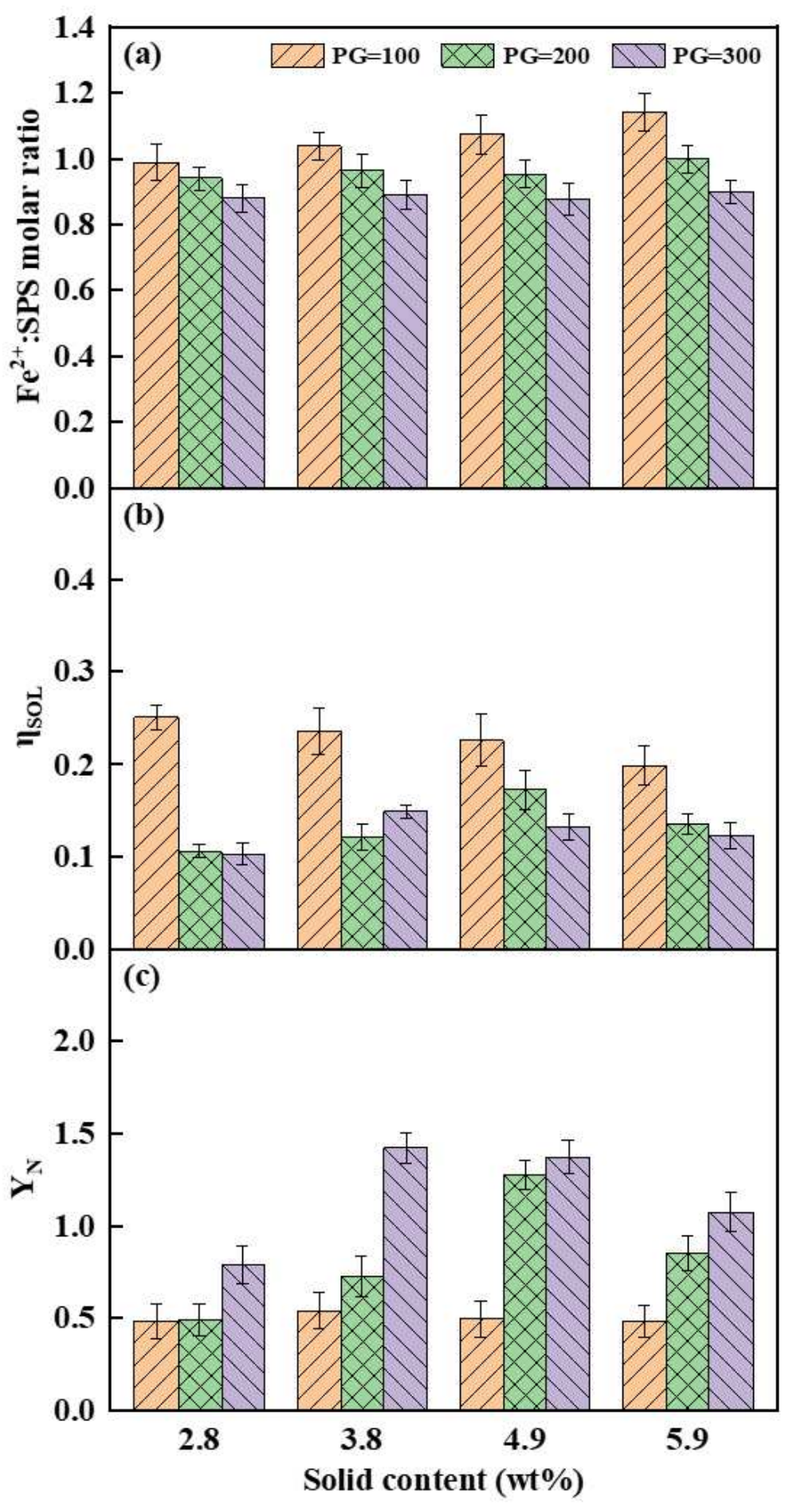

Figure 6

The dynamic relationship between dosage of PG and Fe2+:SPS mol ratio with different solid contents. 


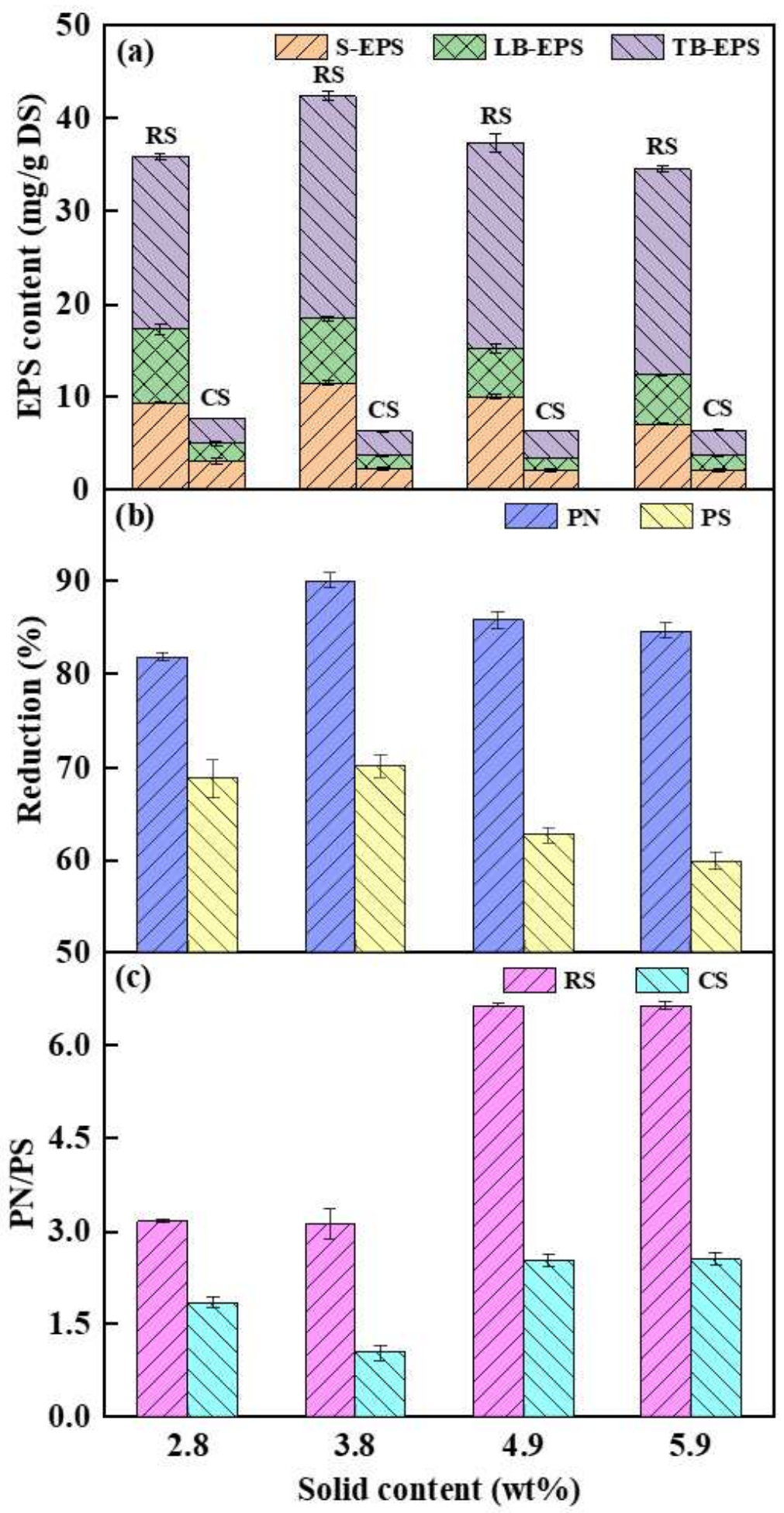

Figure 7

The changes of EPS content (mg/g DS) before and after sludge conditioning. RS $\nabla$ raw sludge; $C S \nabla$ conditioned sludge. TB $\otimes$ tightly bound; LB $\otimes$ loosely bound; $\mathrm{S} \otimes$ slime. PN $\otimes$ protein; $P S \otimes$ polysaccharide.

\section{Supplementary Files}


This is a list of supplementary files associated with this preprint. Click to download.

- Supplementarymaterial.docx 